\title{
On the Combination of SAR and Model Based Techniques for High-Resolution Real-Time Two-Dimensional Reconstruction
}

\author{
Borja Gonzalez-Valdes, Member, IEEE, Yuri Alvarez, Member, IEEE, Jose A. Martinez-Lorenzo, Member, IEEE, \\ Fernando Las-Heras, Member, IEEE, and Carey M. Rappaport, Fellow, IEEE
}

\begin{abstract}
A novel, real-time, two-dimensional method to find the shape of a body from its scattered electric fields is presented. The method is based on a unique combination of the information obtained from the target using the SAR formulation with a novel fast model-based inversion. In the first step, a fast implementation of the SAR inversion is used to recover an initial estimate of the body shape. Secondly, the phase and amplitude of the SAR image are used to extract a set of first guess contours close to the actual position of the body under test. In a third step, these contours are used as a starting point for a model-based inversion in order to disregard the contours that do not match the body profile and to improve the final result. Resolution of less than half of a wavelength for the proposed method is achieved using representative simulation results and measured data
\end{abstract} tion.

Index Terms-Imaging, Inverse Scattering, shape reconstruc-

\section{INTRODUCTION}

I Nverse scattering problems are solved in order to retrieve the shape and constitutive parameters of metallic or dielectric objects from scattered fields measurements. This technique is utilized in a wide range of applications in which noninvasive testing is required, for example, medical diagnostics [1], [2] or detection and identification of buried objects [3] can be cited. Lately, there is also an increasing interest in including this technique in the framework of security scanners for detecting concealed threats in close range [4], [5] or standoff distance [6].

For the latter application, the fast reconstruction of the profile of unknown bodies is of special interest. Diverse techniques to solve this problem, which is in general nonlinear, ill-posed and non-unique, have been proposed. One possibility involves using the so-called inverse source problembased methods [7], [8]. In this approach, a set of equivalent currents within a domain enclosing the object(s)-under-test are reconstructed in order to retrieve the geometry. The scattered

Manuscript received January, 2014. B. Gonzalez-Valdes, J. A. Martinez and C. M. Rappaport are with the ALERT Center of Excellence, Northeastern University, Boston, MA 02115 USA (e-mail: bgonzale@ece.neu.edu; jmartine@ece.neu.edu; rappapor@ece.neu.edu). Borja Gonzalez is also with the Antenna Group at the University of Vigo, 36210 Vigo, Spain. Y. Alvarez, and F. Las-Heras are with the Area of Signal Theory and Communications, Department of Electrical Engineering, Universidad de Oviedo, E-33203, Gijn, SPAIN. Phone: +34-985-182-541; fax: +34-985-182-466 (e-mail: yalopez@ tsc.uniovi.es ; flasheras@tsc.uniovi.es). This work is supported by the U.S. Dept. of Homeland Security under Award Number 2008-ST-061-ED0001, by by the "Ministerio de Economía y Competitividad" of Spain/FEDER under projects CSD2008-00068 (TERASENSE), and TEC2011-24492/TEC (iSCAT) and by the Galician Regional Government under the Plan I2C (2011-2015). fields are related to the currents by a linear or non-linear system of equations [9]. One of the problems arising in these methods is the high computational cost and inversion time, especially in the cases where the observation and reconstruction domains are electrically large. Also, good spatial resolution requires a complicated and costly wideband system.

Other possible approaches are based on the impedance boundary condition [10] or the so-called decomposition methods [11]. Although interesting approaches, these methods still rely on matrix inversions and regularization and do not seem suitable for real time applications.

Another set of techniques solve a nonlinear inverse problem computing iteratively a cost function and minimizing the difference between the field scattered by an evolving contour and the measured data for each inversion iteration. The combination of multiple incidence angles and frequencies improves the accuracy of the reconstruction and overcomes the lack of uniqueness of the solution [12], [13]. This approach yields very good results with limited bandwidth, but it is, in general, computationally intensive as it requires hundreds or thousands of iterations to converge. As a result it becomes impractical for large problems because of the computational cost of the forward methods typically used to calculate the cost function. Recent contributions have reduced the computation time to minutes for simple two dimensional, cylindrical reconstructions, [14] but these techniques are still impractical for applications requiring real-time results.

In this work a Synthetic Aperture Radar (SAR)-based inverse source technique is combined with a model-based inversion to rapidly calculate the shape of a perfect electric conducting (PEC) object. In problems with objects that have slow variation along one dimension a two dimensional analysis is indicated, and will be studied in this paper. The first contour estimation, obtained from the SAR inversion, is used as first guess for the model-based inversion. The use of a fast implementation of the SAR algorithm [15] combined with an efficient model-based inversion [16], [17] allows for high resolution contour reconstruction of electrically large scatterers using very limited bandwidth. In addition, the algorithm can generate real time results. The behavior of the proposed algorithm in a multi-monostatic radar configuration (multiple monostatic radar transceivers imaging the same area) is shown for several application examples using simulated and measured data. 


\section{Methodology Description}

This section presents the methodology used to implement the proposed inversion procedure. Fig 1 presents the vector notation used in the method and a suggested measurement setup for actual implementation of the reconstruction system. Several transmitting-receiving antennas are placed around the object under test in a multi-monostatic configuration. The contour of the object is illuminated from the $p$-th transmitting antenna, which is located at $\boldsymbol{\rho}_{p}$ (where $p=1 . . N_{p}$ and $N_{p}$ is the total number of positions), and then the scattered field is measured in the same observation points.

The method is divided into three major steps. In a first step, an inverse source-based technique [9], accelerated via the inverse Fast Multipole Method (iFMM) [15], is used to retrieve a SAR image of the contour under test. In a second step, the amplitude and phase of the SAR image are combined to extract constant phase lines or phasefronts. In a third step, a model-based inversion, which uses a phase formulation relating the actual measured field and the field scattered by the controlled displacement of a first guess contour, is used [16], [17]. Different first guess contours are selected from the phasefronts extracted in the previous step. The model-based inversion makes it possible to disregard the phasefronts that are placed far from the actual geometric contour of the object, and it provides a better approximation of its actual shape and hence a better reconstruction result.

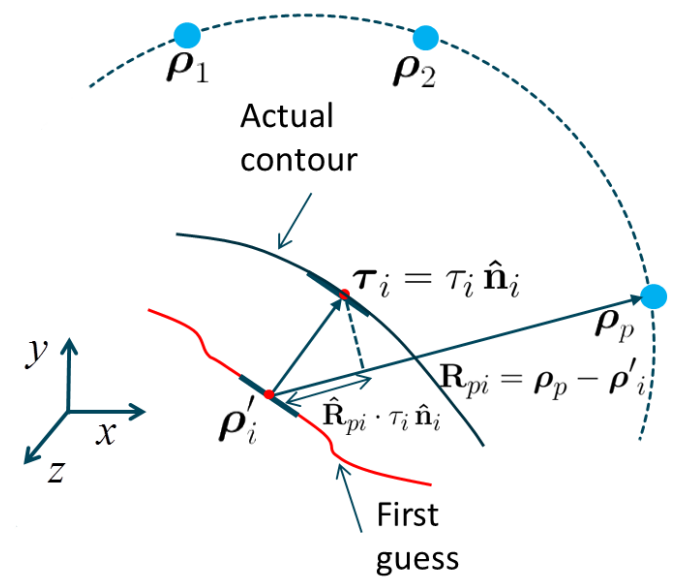

Fig. 1. Top view of the multi-monostratic radar configuration and vectorial formulation for the model based inversion.

\section{A. Synthetic Aperture Radar (SAR) inversion}

Assuming a TM-polarized field (electric field and electric current are oriented in the $\mathrm{Z}$ direction), the electric field scattered by the subdomain $\Omega_{i}$, placed at $\boldsymbol{\rho}_{i}^{\prime}$ in a constant Zplane of the body, at the observation point $\rho_{p}$ can be calculated as [18]:

$$
\mathbf{E}_{i, p}^{s}=\mathbf{E}^{\mathbf{s}}\left(\boldsymbol{\rho}_{p}, \boldsymbol{\rho}_{i}^{\prime}\right)=-\hat{z} \frac{k_{0} \eta}{4} \int_{\Omega_{i}} H_{0}^{(2)}\left(k_{0} \mathbf{R}_{p i}\right) J\left(\boldsymbol{\rho}_{i}^{\prime}\right) d \Omega
$$

where $k_{0}$ and $\eta$ denote the free space wavenumber and impedance, respectively; $H_{0}^{(2)}$ is the Hankel function; $\mathbf{R}_{p i}$ is the distance vector from the observation points to the source points; and $J\left(\boldsymbol{\rho}_{i}{ }^{\prime}\right)$ are the electric currents on the $i$ th subdomain, respectively. The total field at the observation point $\boldsymbol{\rho}_{p}$ can be calculated as:

$$
\mathbf{E}^{\mathbf{S}}\left(\boldsymbol{\rho}_{p}\right)=\mathbf{E}_{p}^{s}=\sum_{i=1}^{N_{\Omega}} \mathbf{E}_{i, p}^{s}
$$

From the scattered field, it is possible to recover an estimate of the equivalent electric currents that radiate the same field as that scattered by the profile under test. The recovered equivalent currents will present maximum amplitude values at the points corresponding to the true profile [9]. Consideration of multiple frequencies will result in several equivalent current distributions that can be coherently combined to provide a SAR image [15]. Using this method, the multi-monostatic SAR image value $I\left(\boldsymbol{\rho}_{u}\right)$ for a pixel placed on $\boldsymbol{\rho}_{u}$, can be calculated as:

$$
I\left(\boldsymbol{\rho}_{u}\right)=\sum_{l, p} \mathbf{E}_{p, l}^{s} e^{+j k_{l} 2 R_{p u}}
$$

where the index $l$ denotes the frequency used with associated wavenumber $k_{l}, \mathbf{E}_{p, l}^{s}$ is the field measured at the frequency $l$ at the observation point $\boldsymbol{\rho}_{p}$ and $R_{p u}$ is the distance between the observation point and the reconstructed pixel.

\section{B. Constant phasefronts extraction}

In a recent publication [19], the possibility of using the phase information contained in the SAR image in order to increase the resolution of the final reconstruction has been suggested. Since the SAR processing is based on backpropagating the received scattered fields, the SAR image constant phasefronts are related to the shape of the profile-under-test. For a radar system with bandwidth $B W$ and center frequency $f_{c}$, the positive frequency response to a scatterer, placed at a distance $r_{o b j}$ from the radar, can be calculated as [20]:

$$
H(f)=\exp \left(-j 2 \pi f \frac{r_{o b j}}{c_{0}}\right)\left[\Pi\left(\frac{f}{B W}\right) * \delta\left(f-f_{c}\right)\right]
$$

where $\Pi\left(\frac{f}{B W}\right)$ defines a frequency window function with bandwidth $B W$. This response corresponds in distance (range) to:

$H(r)=B W \operatorname{sinc}\left(\frac{2 B W}{c_{0}}\left(r-r_{o b j}\right)\right) \exp \left(-j \frac{4 \pi f_{c}}{c_{0}}\left(r-r_{o b j}\right)\right)$

From Eq. (5) the phase of the SAR image, as a function of the position $r$, can be then calculated as:

$$
\phi(r)=-\frac{4 \pi f_{c}}{c_{0}}\left(r-r_{o b j}\right)
$$

Given two points $r_{1}$ and $r_{2}$ whose phase difference is $2 \pi$ in range, $\phi\left(r_{1}\right)-\phi\left(r_{2}\right)=2 \pi$, the distance between $r_{1}$ and $r_{2}$, $\Delta_{r}$, can be calculated as:

$$
\Delta_{r}=\frac{c_{0}}{2 f_{c}}=\frac{\lambda_{c}}{2}
$$


where $\lambda_{c}$ corresponds to the central wavelength of the radar system.

Taking into account the previous relationship, the contour of a body (that can be seen as composed by multiple scatterers) will be reconstructed with a constant, but unknown, phase (see for instance Fig. 4b)). Because of the periodicity of the phase of the backpropagated fields, this phasefront pattern will be periodically repeated according to (7). Unfortunately, it is not possible to directly determine which of the phasefronts is closer to the true profile, and the uncertainty remains about the position of the object inside a certain area, given by the imaging system Point Spread Function (PSF).

The proposed approach for overcoming this limitation requires feeding the phasefronts obtained from the SAR image into a model-based inversion algorithm to find the right position of the scatterer and improve the reconstruction results. To do so, the phasefronts corresponding to points with higher amplitude are selected using an amplitude-based mask to isolate the more relevant phasefronts in the image. Then, linear interpolation is used to create continuous contours (see Fig. 4b)). These contours are finally discretized into facets which are introduced into the model based inversion described in next section.

\section{Model Based Inversion method}

The SAR image provides an initial approximation of the position and shape of the body contour. However, due to the limited bandwidth of the radar system, SAR image resolution may not be sufficient to determine the actual shape of the profile-under-test. Constant phasefronts extracted from the SAR image phase can be used to try to identify the object shape, but as stated in [19], uncertainty about the actual position of the object, among the phasefronts inside the area with higher SAR image amplitude, remains. An iterative model based inversion, described in the flow chart in Fig. 2, is used to obtain the final reconstruction

The obtained phasefronts are sequentially used as a first guess for a Physical Optics model-based inversion [16], [17]. The method is based on an approximation of the nonlinear relationship between the actual scattered field and the one generated by controlled displacements from a first guess contour, as presented in Fig. 1. In previous works, the first guess was estimated from a priori knowledge of the object under test. For the application presented in this paper the following error function is minimized at each frequency [17]:

$$
\begin{gathered}
\mathbf{e}_{p}=\mathbf{E}_{p}^{s, \Delta}-\sum_{i=1}^{N_{\Omega}} \mathbf{E}_{i, p}^{\mathbf{s}} \exp \left(j \phi_{i, p} \tau_{i}\right) \\
\phi_{i, p}=k_{l}\left(-2 \hat{\mathbf{R}}_{p i}\right) \cdot \hat{\mathbf{n}}_{i}
\end{gathered}
$$

where $\mathbf{E}_{p}^{s, \Delta}$ is the field scattered by the actual contour under test a, $\mathbf{E}_{i, p}^{\mathbf{s}}$ is the field radiated according to (1) by the $i$-th facet of the discretized phasefront obtained from the previous step of the method; $\hat{\mathbf{n}}_{i}$ is the normal to the $i$-th facet. The objective of the minimization is to find the values of the vector of distances between the first guess and actual positions of the facets, $\tau_{i}$, that minimizes the euclidean norm of the error function in (8).

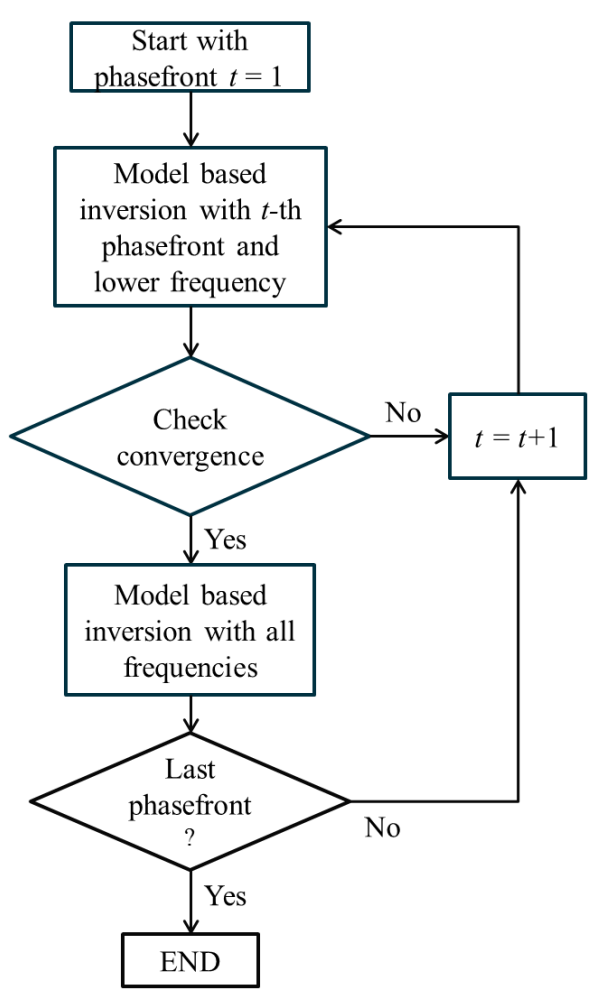

Fig. 2. Flow chart of the model-based inversion method.

In order to solve this non-linear minimization problem, with cost function $\mathbf{e}_{p}$, the Levenberg-Marquardt [17], [21] algorithm is used.

It has been proved in [17] that this minimization only provides convergence to the actual profile when the maximum distortion $\tau_{i}$ is under a quarter of a wavelength of the used frequency, since, for a bigger distortion, the method may get trapped in local minima. The convergence to a local minima is thus detected when the calculated distortions exceed a quarter of a wavelength of the operating frequency. For this application the first guess contours extracted in the previous step are selected to be a quarter of a wavelength apart in range which corresponds to points with phase equal to 0 and $\pi$ radians. This ensures that the distance between at least one of the first guess contours and the actual one is under a quarter of the wavelength at the center frequency. As explained in Fig. 2, the best fit contour is found by checking the convergence after running the model based inversion with the lower available frequency. If the method seems to converge to the actual profile (obtained distortions under a quarter of a wavelenght) the model-based inversion is iteratively performed with the rest of the available frequencies to improve the reconstruction results as stated in [17].

\section{APPLICATION EXAMPLES}

To evaluate the results of the proposed method, three application examples are presented in this section. The first is based on simulated data. To take into account the noise effect, a SNR ratio of $20 \mathrm{~dB}$ is considered by adding white Gaussian noise to the simulated scattered field. In the second example, 
the reconstruction of a measured metal plate is used to prove the behavior of the method with real data. Finally, in the last example, a more complex metallic body is reconstructed to assess the methods performance.

\section{A. Simulation results}

In order to prove the feasibility of the method, the proposed inversion has been first tested using the simulation setup in Fig. 3 to reconstruct the curved Perfect Electric Conductor (PEC) curved contour with two small added distortions (3 $\mathrm{cm}$ square protrusion and $2 \mathrm{~cm}$ square depression) shown. The body geometry is assumed to be invariant along the $\mathrm{Z}$ direction, and the incident field is also polarized in Z. For this configuration and electromagnetic excitation, the problem becomes two-dimensional.

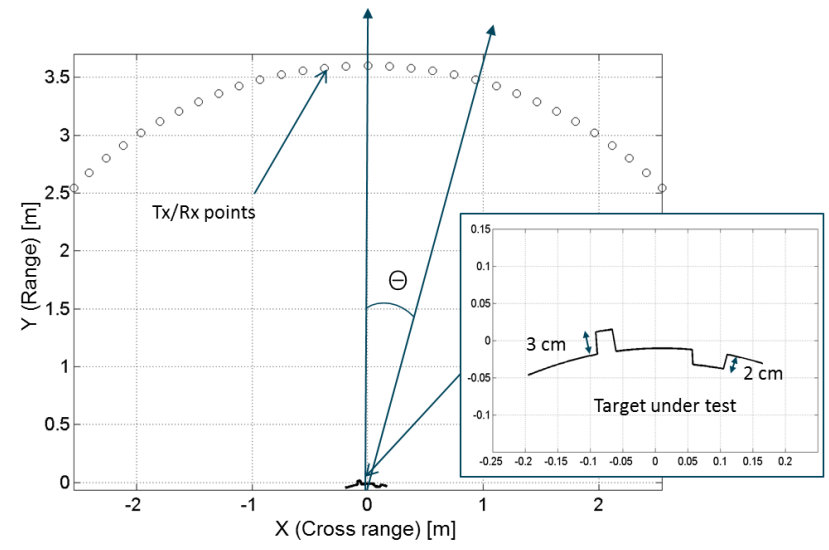

Fig. 3. Setup for the simulation example and zoomed target under test.

The simulation models a monostatic radar configuration with 31 transmitting/receiving points placed on a 120 degrees arc (from $\theta=-60$ to +60 degrees) at $3.6 \mathrm{~m}$. from the target. 10 frequencies are used in this example, from 2.2 to $4 \mathrm{GHz}$ (1.8 GHz bandwidth) with $200 \mathrm{MHz}$ steps. A combination of Method of Moments with the Electric Field Integral Equation (EFIE) is used to compute the synthetic data [18], and both the actual contour and the set of first guess contours, extracted from the phase information of the SAR image, are discretized into segments of size $\lambda / 15$ at the highest frequency (4 GHz).

The extracted first guess contours are also compared with the actual contour under test in Fig. 5. As indicated in Section II, the distance between the 0 and $\pi$ radians phasefronts is around $2.5 \mathrm{~cm}(\lambda / 4$ for the central frequency of $3.1 \mathrm{GHz})$.

Fig. 4 shows the amplitude and phase of the reconstructed SAR image according to Eq. (3). In Fig. 4b), the 0 and $\pi$ radians phasefronts inside the area of the SAR image with normalized amplitude greater than $-15 \mathrm{~dB}$, which are selected as first guess contours for the next step, are shown.

As next step of the method, the 3 obtained contours in Fig. 5 (candidate contours) are used as the first guess in the minimization of the error function in Eq. (8) starting with the lower frequency of $2.2 \mathrm{GHz}$. For the contours \#1 and \#3 in Fig. 5 some of the distortions from the first guess

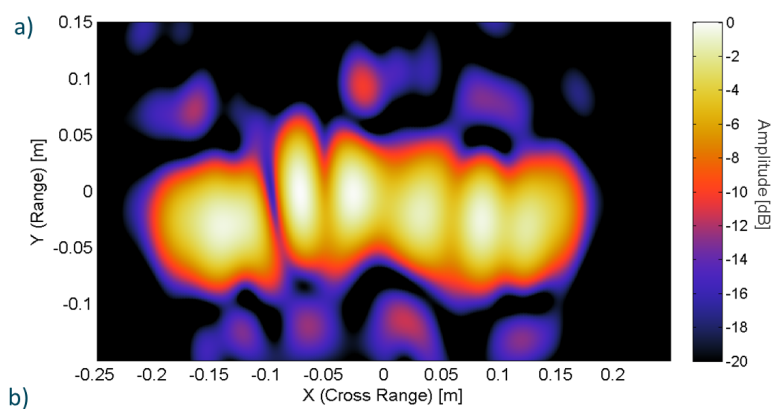

b)

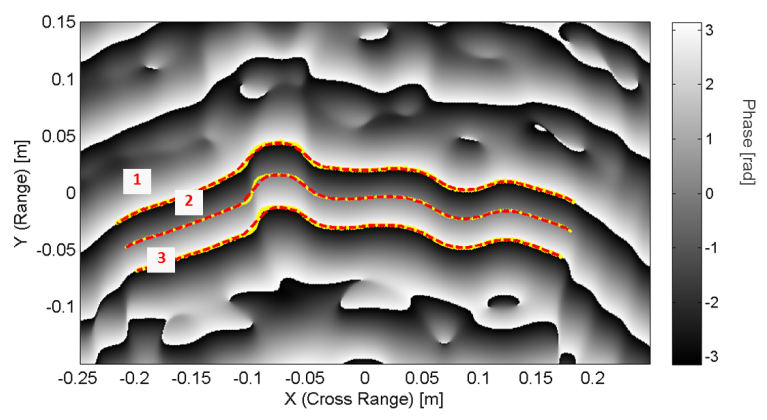

Fig. 4. a) Amplitude of the SAR image from synthesized data of target of Fig. 3 b) Phase with selected phase fronts (yellow dotted lines) and interpolated first guess contours for the model based inversion (dashed red lines).

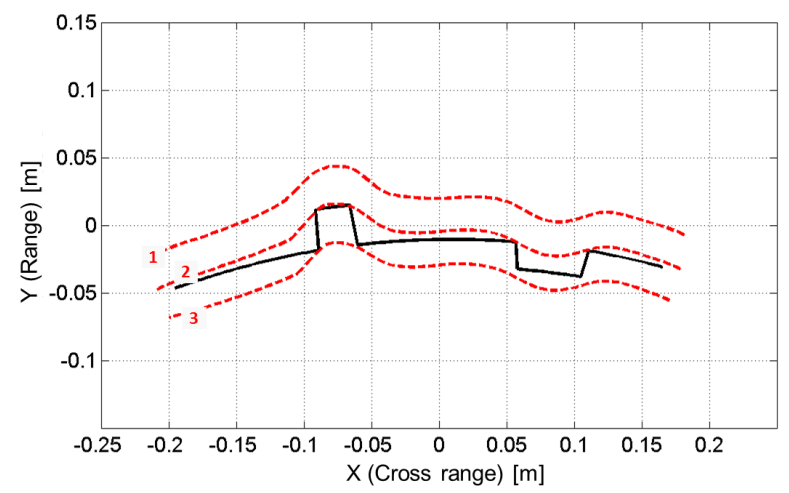

Fig. 5. Actual contour (solid black line) and first guess contours extracted from combining the phase and amplitude of the SAR image (dashed red lines)

calculated by the minimization method are larger than $\lambda / 4$. This demonstrates the convergence of the method to a local minimum with these contours as starting point. For contour \#2 however, the method converges to the contour in the dasheddotted blue line in Fig. 6a). This proves that this contour is closest to the actual one. The successive application of the method for increasing frequencies/iterations as described in [17] yields the reconstruction results at 3 and $4 \mathrm{GHz}$ presented in Figs. 6b) and 6c) respectively.

The reconstruction error is calculated as the Euclidean distance between the points of the actual and recovered profile. Histograms for the first guess contour and final reconstruction errors are presented in Fig. 7. The RMS and maximum errors of the achieved reconstruction are $0.96 \mathrm{~cm}$ and $2.25 \mathrm{~cm}(0.12$ and 0.3 wavelenghts at the higher frequency) respectively. The computational time, which includes the calculation of the MoM synthetic data, SAR processing, phasefronts extraction 


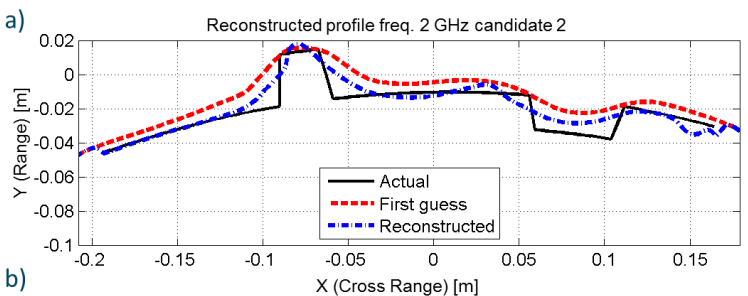

b)
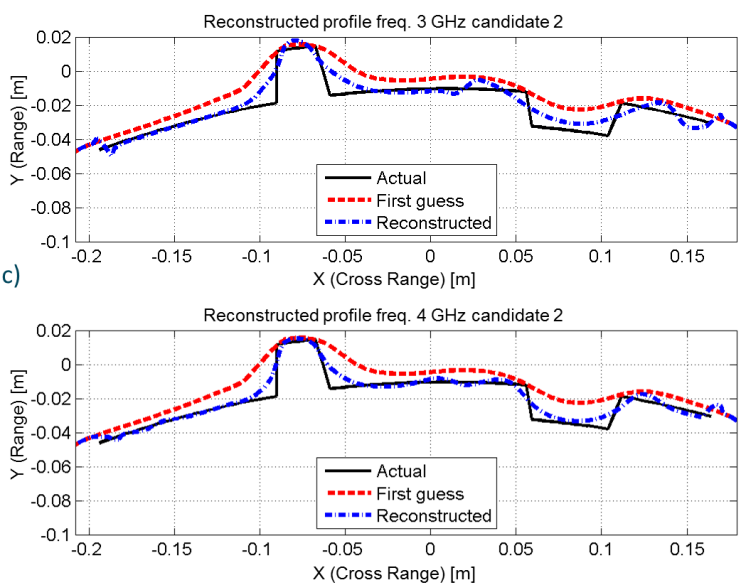

Fig. 6. Actual contour, first guess and reconstructed contour body after applying the model based reconstruction at a) $2.2 \mathrm{GHz}$, b) $3 \mathrm{GHz}$, c) 4 $\mathrm{GHz}$
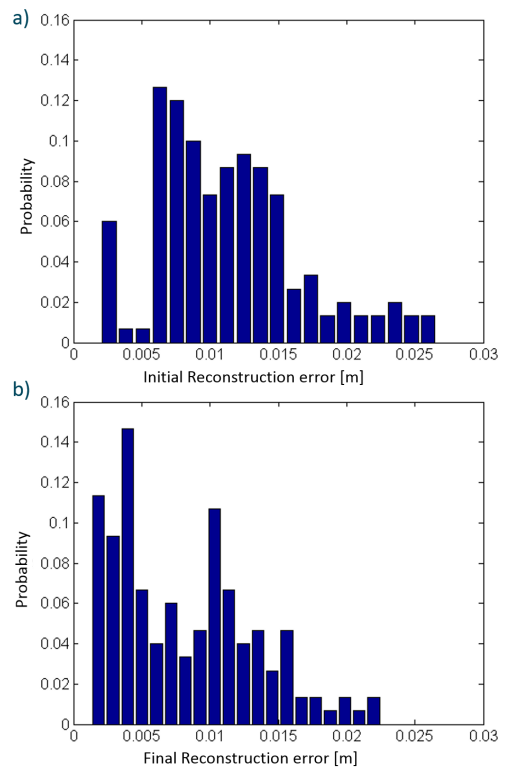

Fig. 7. Histogram of the reconstruction error for a) First guess , b) Final reconstruction after the model based inversion and model based inversion for all the candidates and frequencies, is 8 seconds on a conventional $\mathrm{PC}$ with $3 \mathrm{GHz} \mathrm{CPU}$ clock and 8 GB RAM, using a sequential Matlab code. Both SAR and model-based inversion times can be easily reduced by using CPU-GPU parallel computing [22] applied to the presented approach.

Finally, in order to asses the field convergence, the field scattered by the profile under test, first guess \#2 contour, and the final reconstruction are shown in Fig. 8 in terms of amplitude and phase for $4 \mathrm{GHz}$. The difference between the actual and reconstructed fields is used to asses the overall convergence of the method.
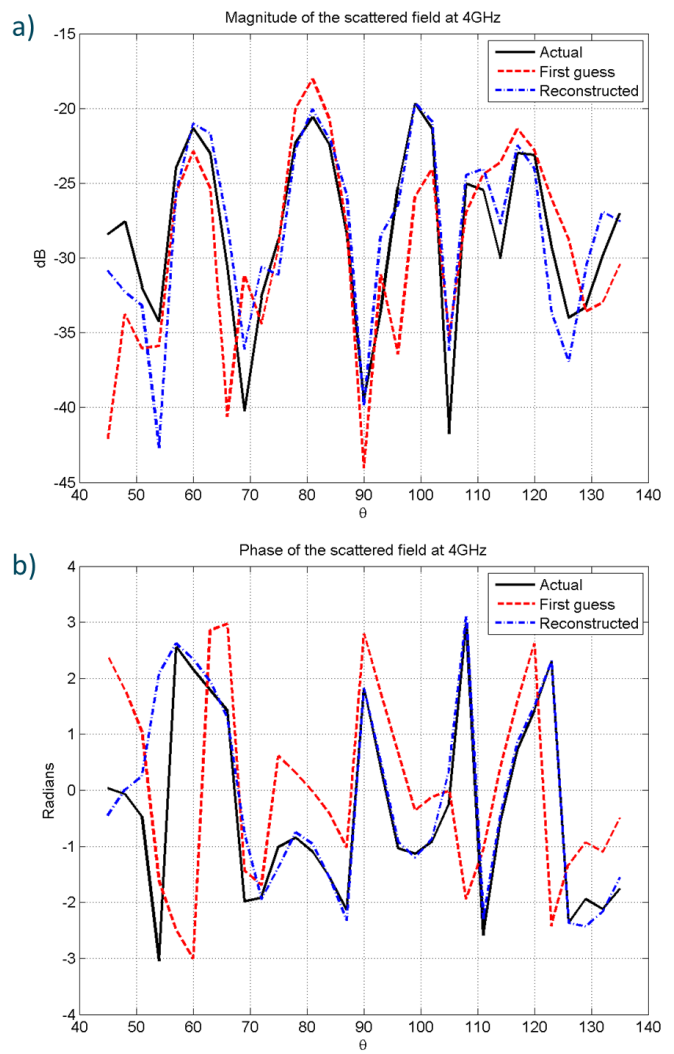

Fig. 8. a) Amplitude and b) phase of the scattered fields at $4 \mathrm{GHz}$ as a function of the observation angle $\theta$ for the simulation example.

\section{B. Validation with Measurements}

In order to experimentally validate the results of the proposed inversion method, measurements were collected at the spherical range in anechoic chamber of the University of Oviedo [23]. The monostatic measurement setup is depicted in Fig. 9.

The field scattered by two different metallic targets has been measured using 31 acquisition points placed on a 120 degrees arc, $3.3 \mathrm{~m}$ away from the object. For this configuration the measurement setup does not allow displacement along the $\mathrm{Z}$ axis, the problem is considered two-dimensional and the contour defined by the cross-section of the object can then be recovered in only one slice. The frequency ranges from 2.4 to $4 \mathrm{GHz}$, in $200 \mathrm{MHz}$-steps. Since both amplitude and phase 


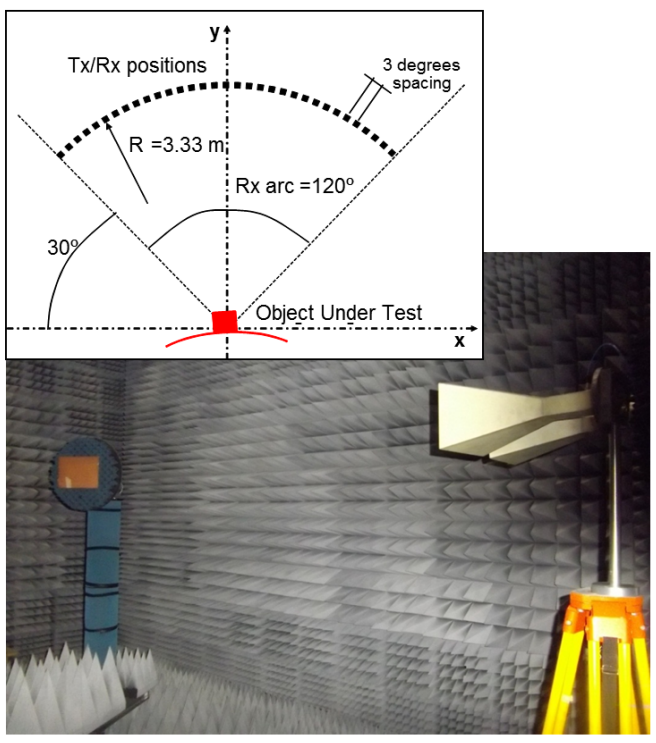

Fig. 9. Measurement setup description and photograph.

are used in the minimization of the error function of Eq. (8), the measured field is normalized in power.

The first measured object consists of a metal plate shown in Fig. 9 which is used to calibrate the method. Fig. 10 shows the amplitude and phase of the reconstructed SAR image. Additionally, in Fig. 10b) the extracted phasefront contours for 0 and $\pi$ radians are superimposed to the phase of the SAR image.

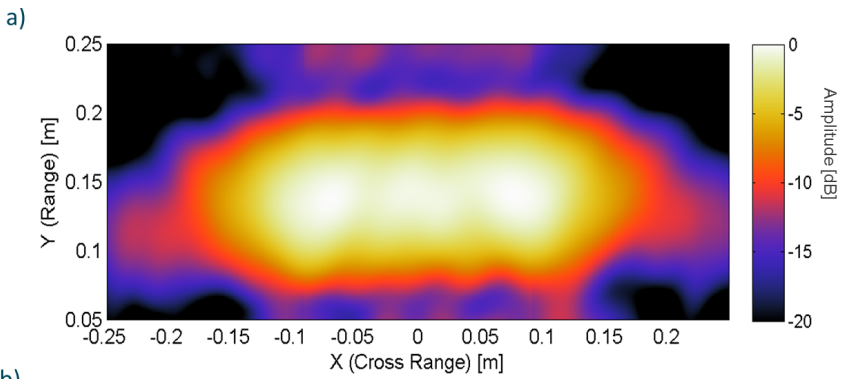

b)

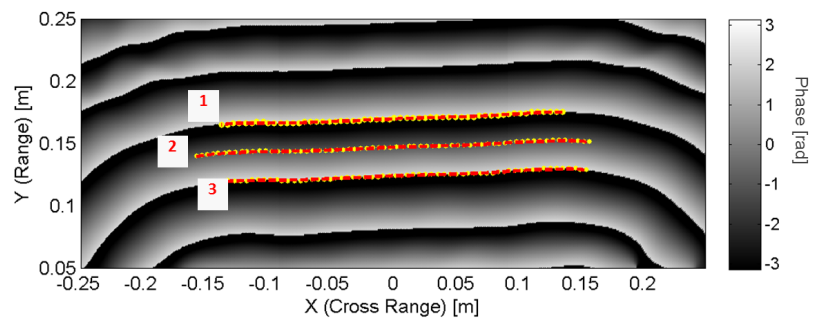

Fig. 10. a) Amplitude of the SAR image of the measured metallic plate. b) Phase with selected phase fronts (yellow dotted lines) and interpolated first guess contours for the model based inversion (dashed red lines).

The extracted phasefronts are then used as first guess in the model-based inversion as stated in previous sections. The convergence of the method to a local minimum allows discarding phasefronts \#2 and \#3 of Fig. 10b) as actual positions for the plate, while the method achieves convergence for the phasefront number 1 whose reconstruction results are
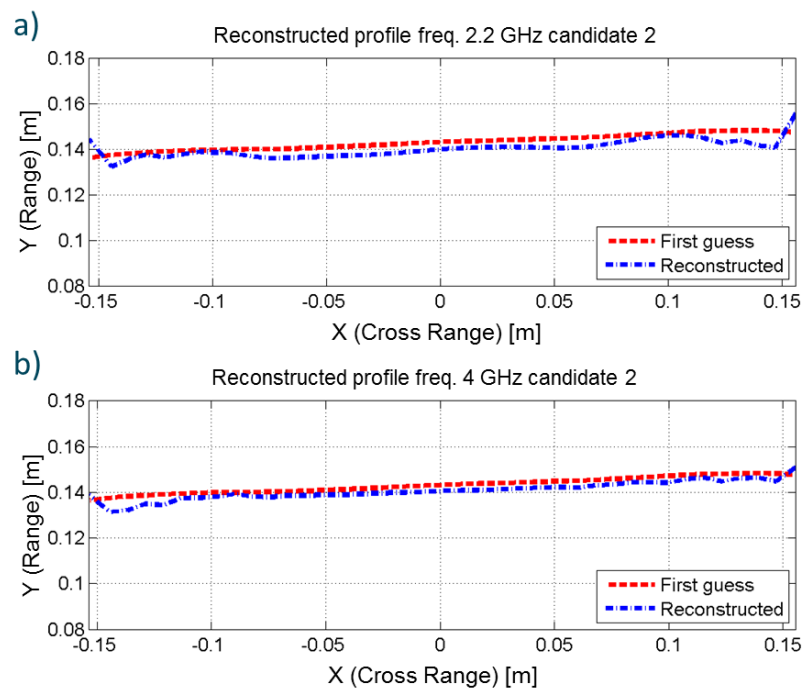

Fig. 11. First guess and reconstructed plate contour after applying the model based reconstruction for a) $2.2 \mathrm{GHz}$, b) $4 \mathrm{GHz}$.

presented in Fig. 11.
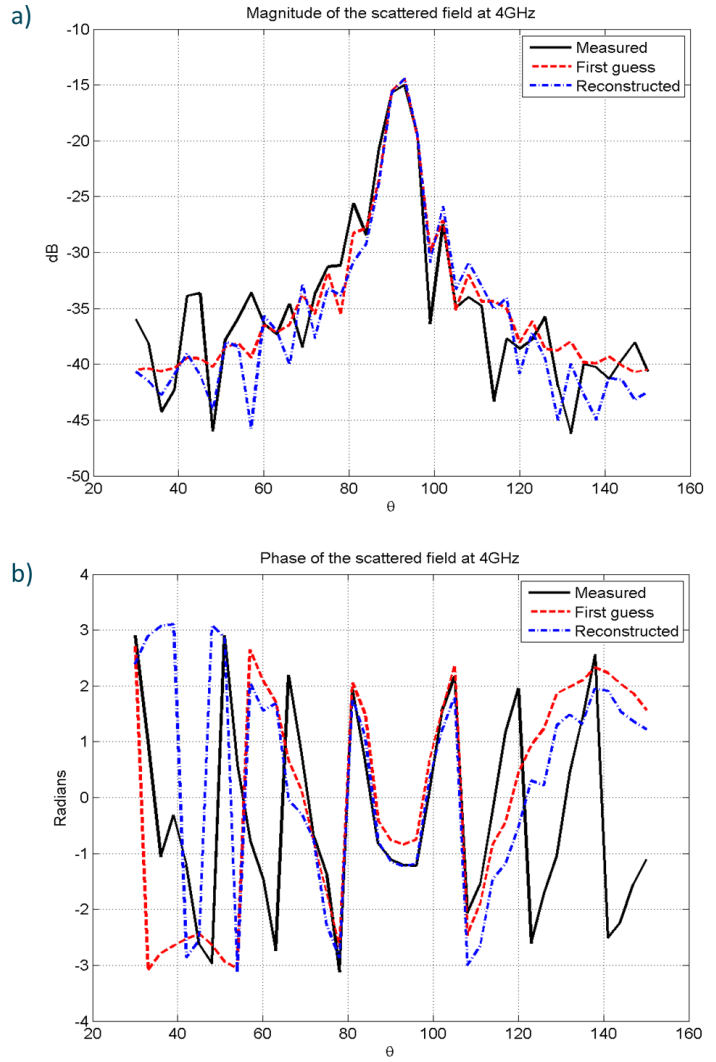

Fig. 12. a) Amplitude and b) phase of the scattered fields at $4 \mathrm{GHz}$ as a function of the observation angle $\theta$ for the measured plate example.

The field convergence for this last case is confirmed in Fig. 12 where the good agreement in the main lobe between reconstructed and measured field in both amplitude and phase is shown.

A curved metallic surface with a $6 \mathrm{~cm}$ thick metallic object (wood covered with aluminum foil) placed vertically 


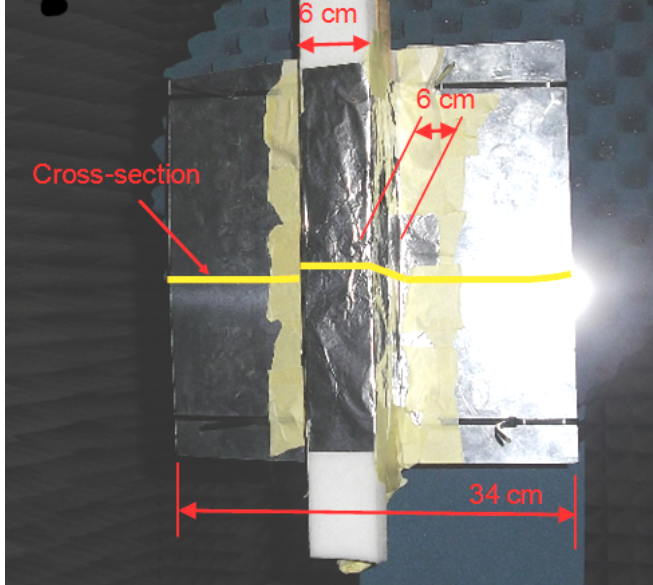

Fig. 13. Photograph of the measured target and cross-section to be reconstruced.

in front of the surface (see Fig. 13) is selected as the second measurement example. As the cross-section of the geometry does not change along elevation axis, the objective is to reconstruct the contour of the object (see the actual crosssection depicted with a solid light yellow profile in Fig. 13).

a)

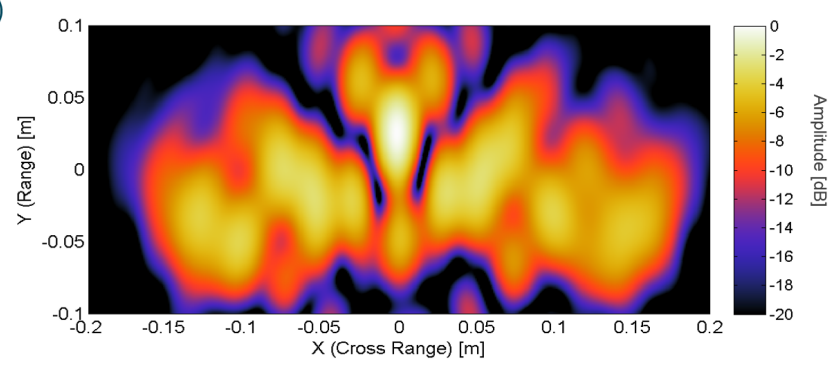

b)

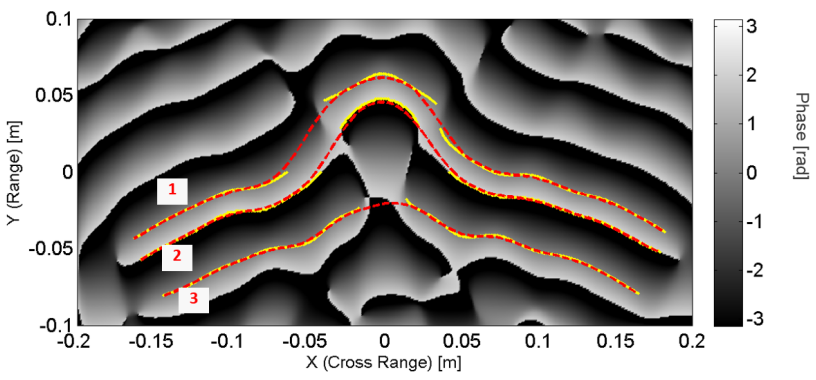

Fig. 14. a) Amplitude of the SAR image of the measured object of Fig 12. b) Phase with selected phase fronts (yellow dotted lines) and interpolated first guess contours for the model based inversion (dashed red lines).

In Fig. 14, the obtained SAR image in amplitude and phase, as well as the extracted phasefronts used in the modelbased inversion step and separated by $\lambda / 4$ at the central frequency, are presented. Due to the nulls in the amplitude, different sections of the phasefronts are isolated and need to be connected during the interpolation process to create a continuous contour.

As in the previous cases, the model-based inversion converges only for one (contour \#2) of the first guess phasefronts. This first guess contour and the final reconstructed profile are presented in Fig. 15. The smooth profile of the curved plate and the $6 \mathrm{~cm}$ square object sticking out from it can be clearly identified after the reconstruction method is applied. Finally, the measured and reconstructed scattered fields are presented in Fig. 16. Good field agreement in both amplitude and phase confirms that the method converges to the right contour. The computational time for both measured examples is about 7 seconds using the same computer described above.
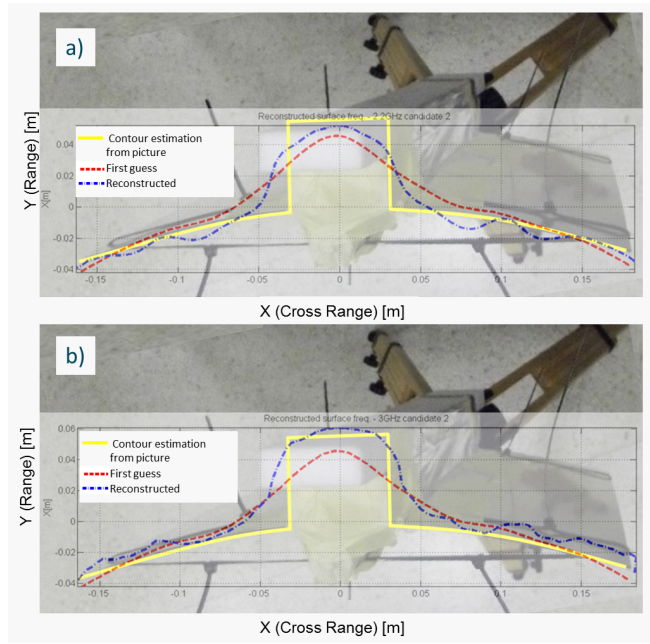

Fig. 15. First guess and reconstructed cross section contour after applying the model based reconstruction for a) $2.2 \mathrm{GHz}$, b) $2.8 \mathrm{GHz}$. The plots are superimposed to a picture of the actual object
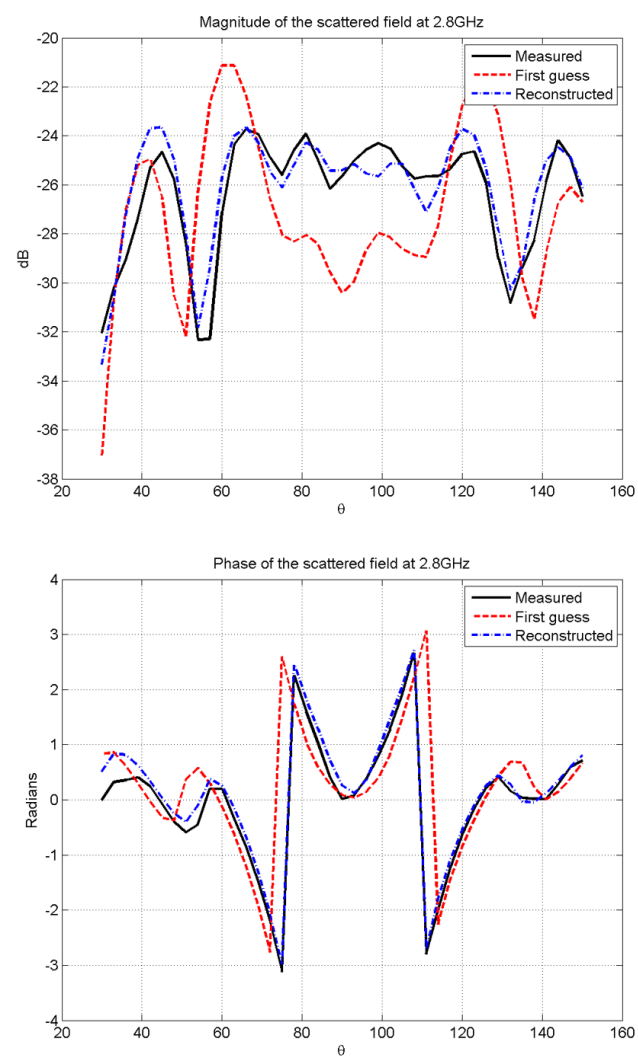

Fig. 16. a) Amplitude and b) phase of the scattered fields at $2.8 \mathrm{GHz}$ as a function of the observation angle $\theta$ for the measured object of Fig. 13 
TABLE I

RECONSTRUCTION PERFORMANCE AS A FUNCTION OF FREQUENCY

\begin{tabular}{|c|c|c|c|c|c|c|c|c|}
\hline $\begin{array}{l}\text { Freq. range } \\
{[\mathrm{GHz}]}\end{array}$ & $\begin{array}{l}\text { Observation } \\
\text { points }\end{array}$ & Candidates & $\begin{array}{l}\text { SAR } \\
\text { time }[s]\end{array}$ & $\begin{array}{l}\text { Phasefronts ex- } \\
\text { traction time [s] }\end{array}$ & $\begin{array}{l}\text { Model-based } \\
\text { inv. time [s] }\end{array}$ & Total time $[\mathrm{s}]$ & $\begin{array}{l}\text { Max. error } \\
{[\mathrm{cm}]}\end{array}$ & $\begin{array}{l}\text { Max error } \\
{[\lambda]}\end{array}$ \\
\hline $1-2$ & 48 & 3 & 0.28 & 0.01 & 4.53 & 4.82 & 1.621 & 0.108 \\
\hline $2-3$ & 72 & 5 & 0.42 & 0.02 & 7.16 & 7.60 & 0.869 & 0.087 \\
\hline $3-4$ & 96 & 7 & 0.30 & 0.03 & 8.37 & 8.71 & 0.665 & 0.089 \\
\hline $4-5$ & 120 & 9 & 0.39 & 0.03 & 9.82 & 10.24 & 0.598 & 0.100 \\
\hline $5-6$ & 144 & 11 & 0.49 & 0.04 & 13.25 & 13.78 & 0.484 & 0.097 \\
\hline $6-7$ & 168 & 13 & 0.55 & 0.06 & 25.40 & 26.00 & 0.386 & 0.090 \\
\hline $7-8$ & 192 & 14 & 0.72 & 0.07 & 28.78 & 29.57 & 0.346 & 0.092 \\
\hline $8-9$ & 216 & 16 & 0.79 & 0.10 & 34.77 & 35.65 & 0.264 & 0.079 \\
\hline $9-10$ & 240 & 18 & 0.98 & 0.10 & 40.77 & 41.84 & 0.253 & 0.084 \\
\hline $10-11$ & 265 & 19 & 1.23 & 0.13 & 54.14 & 55.50 & 0.211 & 0.078 \\
\hline
\end{tabular}

\section{PARAMETRIC STUDY}

In order to assess the performance of the proposed method as a function of the electric size of the target, a parametric study has been performed. The profile-under-test is $2 \mathrm{~m}$ long with $10 \mathrm{~cm}$ roughness. The true profile, which is similar to the example presented in [24], is shown in Fig. 17. The presented method has been tested using $1 \mathrm{GHz}$ bandwidth centered around ten different frequencies ranging from 1.5 to $10.5 \mathrm{GHz}$ (the electrical size of the object varies from approximately 13 to 73 wavelengths). The observation points are placed in an arc ( $\mathrm{R}=4.6 \mathrm{~m}$ ), sampled every one wavelength, so the number of observation points increases with the frequency. Six frequency steps are used $\left(\Delta_{f}=200 \mathrm{MHz}\right)$ in all cases noting that the number of frequencies needed to prevent aliasing depends only on the bandwidth. The code was run on a workstation equipped with 12 CPUs @ $3.15 \mathrm{GHz}$, with 128 GB RAM, but only one CPU without parallel processing was used for this.

Table I summarizes the results of the study and specifies the time consumed in each step of the method. Note that the method spends most of its time on the model based inversion for the different first guess contours obtained from the phasefronts. The number of candidate phasefronts increases with frequency because the phasefronts are closer, according to (7). In the current implementation of the method, the different contours are sequentially evaluated with the model based inversion. However, it would be possible to evaluate the different contours in parallel, thus reducing further the processing time. As an example, the application of the model based inversion to the contour that converges for the frequency range of 10 to $11 \mathrm{GHz}$ takes around 6 seconds. If the evaluation of the different contours were implemented in parallel, the total processing time would be reduced from 54 to around 7.5 seconds (SAR time, phasefront extraction and time for converging phasefront) for the presented electrically large problem (73 wavelengths in the $10-11 \mathrm{GHz}$ frequency band). Fig. 17 presents the actual, first guest and reconstructed profiles for the highest and lowest frequencies considered in the study.

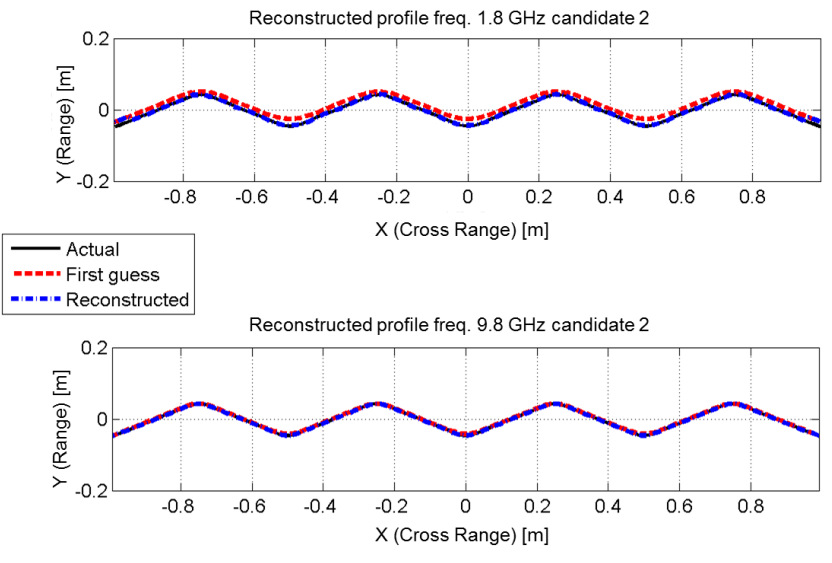

Fig. 17. Reconstruction results for a) $1-2 \mathrm{GHz}$ b) $9-10 \mathrm{GHz}$.

\section{CONCLUSIONS}

In this paper a fast novel technique for two-dimensional contour reconstruction has been presented. This approach combines the advantages of SAR and model-based inversion to achieve real time high resolution reconstruction results with small bandwidth (central frequency of $3.2 \mathrm{GHz}$ and $1.6 \mathrm{GHz}$ of BW). The use of the phase and amplitude of the SAR based inversion provides a good first guess for the model-based method, allowing for fast, robust and accurate convergence to the contour of the object under test. Resolution below half a wavelength for the proposed approach has been demonstrated through alternate simulation and measurement results. The method is able to approximate sharp profiles as small as 0.4 wavelengths, although representing them as smooth profiles due to the use of low frequencies. Also, a parametric study to assess the processing time as a function of the electric size of the target has been presented, showing that the method can provide real time capabilities (assuming parallel calculation) even for electrically large problems. Further work will be related to the extension of the method for $3 \mathrm{D}$ problems where objects under test present structural variance in the $\mathrm{Z}$ dimension. 


\section{ACKNOWLEDGMent}

The authors would like to acknowledge S. Rappaport for her suggestions and corrections regarding the readability of the manuscript.

\section{REFERENCES}

[1] A. T. Vouldis, C. N. Kechribaris, T. A. Maniatis, K. S. Nikita, and N. K. Uzunoglu, "Investigating the enhancement of three-dimensional diffraction tomography by using multiple illumination planes," $J$. Opt. Soc. Am. A, vol. 22, no. 7, pp. 1251-1262, Jul 2005.

[2] E. Fear, X. Li, S. Hagness, and M. Stuchly, "Confocal microwave imaging for breast cancer detection: localization of tumors in three dimensions," Biomedical Engineering, IEEE Transactions on, vol. 49, no. 8, pp. 812-822, 2002

[3] J. Martinez-Lorenzo, C. Rappaport, and F. Quivira, "Physical limitations on detecting tunnels using underground-focusing spotlight synthetic aperture radar," Geoscience and Remote Sensing, IEEE Transactions on, vol. 49, no. 1, pp. 65-70, 2011.

[4] D. Sheen, D. McMakin, and T. Hall, "Three-dimensional millimeterwave imaging for concealed weapon detection," Microwave Theory and Techniques, IEEE Transactions on, vol. 49, no. 9, pp. 1581-1592, 2001.

[5] X. Zhuge and A. Yarovoy, "A sparse aperture mimo-sar-based uwb imaging system for concealed weapon detection," Geoscience and Remote Sensing, IEEE Transactions on, vol. 49, no. 1, pp. 509-518, 2011.

[6] K. Cooper, R. Dengler, N. Llombart, B. Thomas, G. Chattopadhyay, and P. Siegel, "Thz imaging radar for standoff personnel screening," Terahertz Science and Technology, IEEE Transactions on, vol. 1, no. 1, pp. 169-182, 2011.

[7] M. Pastorino, A. Massa, and S. Caorsi, "A microwave inverse scattering technique for image reconstruction based on a genetic algorithm," Instrumentation and Measurement, IEEE Transactions on, vol. 49, no. 3 , pp. 573-578, 2000.

[8] S. Caorsi, G. Gragnani, and M. Pastorino, "Two-dimensional microwave imaging by a numerical inverse scattering solution," Microwave Theory and Techniques, IEEE Transactions on, vol. 38, no. 8, pp. 981-980, 1990.

[9] Y. Alvarez Lopez, A. Dominguez-Casas, C. Garcia-Gonzalez, and F. Las-Heras, "Geometry reconstruction of metallic bodies using the sources reconstruction method," Antennas and Wireless Propagation Letters, IEEE, vol. 9, pp. 1197-1200, 2010.

[10] G. S. Ünal, M. Çayören, and E. Tetik, "Reconstruction of surface impedance of an object located over a planar pec surface," Journal of Physics: Conference Series 135, 2008.

[11] Y. A. Godin and B. Vainberg, "The inverse scattering problem for the difference operators: the decomposition method," Journal of Physics: Conference Series 135, vol. 1 ISSN, 2008.

[12] M. R. Hajihashemi and M. El-Shenawee, "Shape reconstruction using the level set method for microwave applications," Antennas and Wireless Propagation Letters, IEEE, vol. 7, pp. 92-96, 2008.

[13] M. E.-S. Mohammad R. Hajihashemi, "Inverse scattering of threedimensional pec objects using the level-set method," Progress in Electromagnetic Research, vol. 116, pp. 23-47, 2011.

[14] A. M. Hassan, T. Bowman, and M. El-Shenawee, "The linear sampling method for the acceleration of the level set algorithm," in Antennas and Propagation Society International Symposium (APSURSI), 2012 IEEE, july 2012, pp. $1-2$.

[15] Y. Alvarez, J. Martinez, F. Las-Heras, and C. Rappaport, "An inverse fast multipole method for imaging applications," Antennas and Wireless Propagation Letters, IEEE, vol. 10, pp. 1259 -1262, 2011.

[16] J. Martinez-Lorenzo, B. Gonzalez-Valdes, C. Rappaport, and A. Pino, "Reconstructing distortions on reflector antennas with the iterativefield-matrix method using near-field observation data," Antennas and Propagation, IEEE Transactions on, vol. PP, no. 99, p. 1, 2011.

[17] B. Gonzalez-Valdes, J. A. Martinez-Lorenzo, and C. M. Rappaport, "A new fast algorithm for radar-based shape reconstruction of smoothly varying objects," IEEE Antennas and Wireless Propagation Letters, vol. 12 , pp. $484-487,2013$.

[18] A. F. Peterson, S. L. Ray, and R. Mittra, Computational Methods for Electromagnetics,. The IEEE/OUP Series on Electromagnetic Wave Theory and Oxford University Press, 1997.

[19] Y. Alvarez, B. Gonzalez-Valdes, J. Martinez, F. Las-Heras, and C. Rappaport, "An improved sar based technique for accurate profile reconstruction," IEEE Transactions on Antennas and Propagation, vol. 61, no. 3, pp. 1490-1495, 2013.
[20] D. K. Barton, A. I. Leonov, I. A. Morozov, and P. C. Hamilton, Radar technology Encyclopedia. Artech House, 1996.

[21] D. W. Marquardt, "An algorithm for least-squares estimation of nonlinear parameters," Siam Journal on Applied Mathematics, vol. 11, 1963.

[22] L. E. Tirado, J. A. Martinez-Lorenzo, B. Gonzalez-Valdes, C. Rappaport, O. Rubinos-Lopez, and H. Gomez-Sousa, "GPU implementation of the modified equivalent current approximation (MECA) method," Applied computational electromagnetics society journal, no. 9, SEP 2012.

[23] Y. Alvarez, M. Elena de Cos, and F. Las-Heras, "RCS measurement setup for periodic-structure prototype characterization," IEEE Ante, vol. 52, no. 3, pp. 100-106, Jun 2010.

[24] S. Kidera, T. Sakamoto, and T. Sato, "Super-resolution UWB radar imaging algorithm based on extended capon with reference signal optimization," Antennas and Propagation, IEEE Transactions on, vol. 59, no. 5, pp. 1606-1615, 2011.

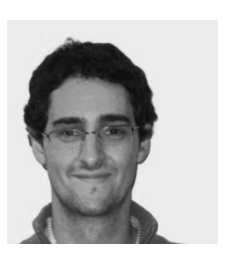

Borja Gonzalez-Valdes (S'07-M'10) received the B.S and Ph.D. in Electrical Engineering from the University of Vigo, Spain, in 2006 and 2010 respectively. From 2006 to 2010 he was with the Antenna and Optical Communications group at the University of Vigo. During 2008 and 2009 he was a visiting researcher at the Gordon CenSSIS, Northeastern University, Boston, USA. In 2011 he joined the ALERT Center of Excellence, Northeastern University, Boston, USA. His research interests include imaging techniques, and $\mathrm{THz}$ technology.

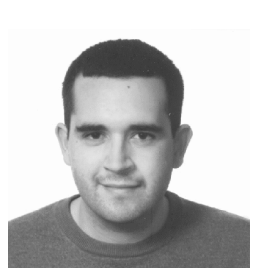

Yuri Alvarez (S'06-M'09) was born in Langreo (Spain), in 1983. He received the M.S. and Ph.D. degree in Telecommunication Engineering in 2006 and 2009 from the University of Oviedo. He was a Visiting Scholar at the Dept. of Electrical Eng. and Computer Science, Syracuse University, Syracuse (USA) in 2006 and 2008; and a Visiting Postdoc at the Gordon Center for Subsurface Sensing \& Imaging Systems (CenSSIS) ALERT Center of Excellence, Northeastern University, Boston (USA) from 2011 to 2013. He is currently an Assistant Professor at the Signal Theory and Communications of the University of Oviedo (Gijn, Spain). He received the 2011 Regional and National Awards to the Best Ph.D. Thesis on Telecommunication Engineering (category: security and defense). His interests and research studies have been focused on antenna diagnostics, antenna measurement techniques, RF techniques for indoor location, and inverse scattering and imaging techniques. 


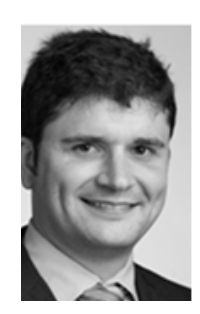

Prof. Jose A. Martinez-Lorenzo (S'03-M'05) received the B.S/M.S. degree in 2002 and the Ph.D. degree in 2005, both in electrical engineering from the University of Vigo, in Spain. He joined the faculty at University of Oviedo in Gijon, Spain in 2004, where he was an Assistant Professor in the Department of Signal Theory and Communications. In 2006, he joined Bernard M Gordon Center for Subsurface Sensing and Imaging Systems, at Northeastern University. In 2010 he was appointed as a Research Assistant Professor in the ECE Department at Northeastern. Since August 2013, he holds a joint appointment in the Departments of MIE and ECE as a Tenure-Track Assistant Professor. He is an active member of ALERT (Awareness and Localization of Explosives-Related Threats) a DHS Center of Excellence awarded to Northeastern. Prof. Jose A. Martinez-Lorenzo has authored over 140 technical journal and conference papers. His research is geared towards the understanding, modeling and solving complex engineering problems, with an emphasis on mechanical and electromagnetic sensing and imaging methods for security and biomedical applications (i.e.- explosive detection, breast cancer detection). He led the team that won the best paper award, in the 2012 IEEE Conference on Technologies for Homeland Security, for the paper on A compressed sensing approach for detection of explosive threats at standoff distances using a Passive Array of Scatterers.

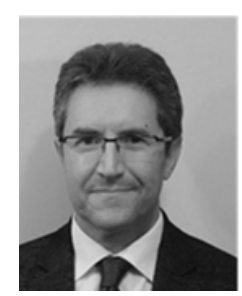

Fernando Las-Heras (M'86SM'08) received the M.S. degree in 1987 and the Ph.D. degree in 1990, both in Telecommunication Engineering, from the Technical University of Madrid (UPM), Spain. From 1988 to 1990 , he was a National Graduate Research Fellow. From 1991 to 2000 he held a position of Associate Professor at the Department of Signal, Systems and Radiocommunications of the UPM. From December 2003 he holds a Full-Professor position at the University of Oviedo where he has been Vice-dean for Telecommunication Engineering at the Technical School of Engineering at Gijón from 2004 to 2008. From 2001 he heads the research group Signal Theory and Communications TSCUNIOVI at the Dept. of Electrical Engineering of the University of Oviedo, being the principal investigator in several projects of national programs and contracts with companies. He was Visiting Lecturer at the National University of Engineering in Peru in 1996, Visiting Researcher at Syracuse University, New York, in 2000, and short term Visiting Lecturer at ESIGELEC in France since 2005. From 2005 he holds the Telefonica Chair ICTs applied to Environment and Climate Change in the University of Oviedo and from 2010 he is member of the Science, Technology and Innovation Council of Asturias, Spain. He has authored over 300 technical journal and conference papers in the areas of antenna design, the inverse electromagnetic problem with application to diagnostic, measurement and synthesis of antennas, propagation and computational electromagnetics and engineering education.

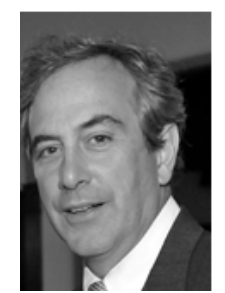

Carey M. Rappaport (IEEE M, SM'96, F'06) received five degrees from the Massachusetts Institute of Technology: the SB in Mathematics, the $\mathrm{SB}, \mathrm{SM}$, and EE in Electrical Engineering in June 1982, and the PhD in Electrical Engineering in June 1987. He is married to Ann W. Morgenthaler, and has two children, Sarah and Brian. Prof. Rappaport has worked as a teaching and research assistant at MIT from 1981 until 1987, and during the summers at COMSAT Labs in Clarksburg, MD, and The Aerospace Corp. in El Segundo, CA. He joined the faculty at Northeastern University in Boston, MA in 1987. He has been Professor of Electrical and Computer Engineering since July 2000. In 2011, he was appointed College of Engineering Distinguished Professor. During fall 1995, he was Visiting Professor of Electrical Engineering at the Electromagnetics Institute of the Technical University of Denmark, Lyngby, as part of the W. Fulbright International Scholar Program. During the second half of 2005, he was a visiting research scientist at the Commonwealth Scientific Industrial and Research Organisation (CSIRO) in Epping Australia. He has consulted for CACI, Alion Science and Technology, Inc., Geo-Centers, Inc., PPG, Inc., and several municipalities on wave propagation and modeling, and microwave heating and safety. He was Principal Investigator of an ARO-sponsored Multidisciplinary University Research Initiative on Humanitarian Demining, Co-Principal Investigator of the NSF-sponsored Engineering Research Center for Subsurface Sensing and Imaging Systems (CenSSIS), and Co-Principal Investigator and Deputy Director of the DHS-sponsored Awareness and Localization of Explosive Related Threats (ALERT) Center of Excellence. Prof. Rappaport has authored over 400 technical journal and conference papers in the areas of microwave antenna design, electromagnetic wave propagation and scattering computation, and bioelectromagnetics, and has received two reflector antenna patents, two biomedical device patents and four subsurface sensing device patents. He was awarded the IEEE Antenna and Propagation Society's H.A. Wheeler Award for best applications paper, as a student in 1986. He is a member of Sigma Xi and Eta Kappa Nu professional honorary societies. 